International Journal of Engineering \& Technology, $7(2.17)(2018) 95-100$
International Journal of Engineering \& Technology
SPC
Website: www.sciencepubco.com/index.php/IJET
Research Paper

\title{
Novel Sgmentation Technique for Synthetic Aperture Radar Target Tracking using Hybrid PSO Method
}

\author{
B. Malakonda Reddy, Md Zia Ur Rahman* \\ Department of Electronics and Communication Engineering, Koneru Lakshmaiah Education Foundation, Green fields, \\ Guntur DT, AP, 522502, India. \\ *Corresponding author E-mail:mdzr55@gmail.com
}

\begin{abstract}
In remote sensing applications, it is evidently electromagnetic imaging has more advantages than optical imaging due to its horizon. In such a contest synthetic aperture radars (SAR) plays a vital role. In SAR image processing, segmentation is a key step in identifying and tracking targets, terrain features. Hence, this paper, we present an Improved hybrid PSO method proposed based on multilevel threshold for enhancing the image for segmentation. Experimental results indicate, the proposed methods enhance the edge features effectively with compare to Otsu, Modified Otsu and Region Based Active contour methods.
\end{abstract}

Keywords: Electromagnetic Imaging, synthetic aperture radars, remote sensing, terrain features, segmentation methods.

\section{Introduction}

Synthetic aperture radar inherently suffers from speckle noise because of the coherent imaging system. However, multi look averaging is the general way to remove the speckle noise at the cost of reduced spatial resolution. Speckle noise is increasing in nature that ends up in face the difficulties within the analysis of pictures by degrading the sides and tiny disorders within the image. As a result poor quality image is feasible. It'sa lot of favorable to develop appropriate filtering techniques. Though the classical filters love Frost filter[1],Lee filter[2], and Kuan filter[3] that denoise SAR pictures in the special domain by recalculating the middle pixels of the filtering windows supported the native scene heterogeneousness, work well in stationary image space, they have a tendency to either preserve speckle noise or erase a weak scene signal. This necessitates economical techniques to boost the image and segregate the regions within the Image. Once segregating the image we will notice the quantity of objects or special regions within the Image. The manual detection is the complicated task to distinguish the similarly or distinctive region in the image, it makes the stress on the human eye. In order to overcome the problem in image capture and manual detection an enhanced system with denoising filters and segmented algorithm is proposed.

In this paper, several methods for speckle noise reduction have been proposed. In present days the effective way of denoising technique is based on wavelet threshold techniques. But it has a high computational cost. [4] -[8].Sforza et al. proposed an adaptive threshold technique to detect the gray area ofa noisy image. Automatic segmentation is proposed by Delsanto et al. For artery segmentation. Geo et al. Proposed anoptimal threshold method for image segmentation based optical swarm optimization [9].Region growing methods increases reorganizationrate, but less sensitive to noise. Giordano et.al and Osowski proposed efficient preprocessing techniques for image processing [10]. Image enhancement using multi frequency techniques was introduced by Casciaro et al. After that segmentation based on the active contour method is introduced by ChangandVese using level set methods.

\section{Electromagnetic SAR Image Segmentation}

In SAR image, segmentation is one of the difficult tasks to extract the objects or region in blurred image because of speckle noise. As discussed earlier the denoised image is taken as input to segmentation. The commonly used method is Otsu method, it binaries the image based on threshold. It divides the image into groups based on the principle of minimization of class variables. But Otsu method does not produce the most accurate results. After for the better result modified Otsu is introduced by the calculation of new threshold [11]-[13].

The threshold value for the single iteration is calculated as

$$
T_{h 1}=\frac{\mu_{1}^{[1]}+\mu_{2}^{[1]}}{2}
$$

The new threshold value $T_{h 2}$ is calculated based on previous threshold value $T_{h 1} . \mu_{1}$ and $\mu_{2}$ represents the mean values above and below the current threshold respectively. The total iteration process continues until the threshold value converges to

$T^{\prime}=\frac{\mu_{1}^{[k]}+\mu_{2}^{[k]}}{2}$

The mean is calculated for the whole image and is given as $\mu_{n}=$ $\sum_{i=1}^{s} i \operatorname{Pr}(i)$

It is observed that the threshold values for modified Otsu produce less than the conventional Otsu method after making the experiments on different images. In order to further increment the accuracy another technique was introduced based on region growing active contour technique. Several image processing, signal processing and array processing techniques for SAR imaging are presented in [32]-[42].

\subsection{Hybrid Region Based Active Contour Method}

In region based segmentation contour initialization is a difficult task [14]-[18]. The contour can move away from the 
object if initial initialization is bad in condition. The level set technique produces remedy for this by manual placing of contour for segmentation. This paper presents the hybrid method that combines the region based segmentation and modified Otsu method for automatic segmentation of regions. In the proposed hybrid technique, the contour initializes the binary mask of black and white regions which is produced by modifying Otsu method. By this method contour occupies the object boundary very quickly. The initial contour can be placed using level set function $\Psi$

The initial contour is represented as $C^{\prime}=\{(y, z), \Psi(y, z)\}=0$ The level set function $\Psi$ is updated and exist wwithinthe narrow band and the computational time taken by this level set function is reduced by this narrow band approach. The narrow band equation is given byeq (4)

$$
\dot{C}_{N B}=(m, n)-\beta_{0} \leq \beta_{0}, m=[0, i-1], n=[0, j-1]
$$

The level set function mean curvature is given as

$$
\text { Mean curvature }=\frac{\left(\Psi_{y}^{2} * \Psi_{z z}+\Psi_{z}^{2} * \Psi_{y y}-2 \Psi_{y} \Psi_{z} \Psi_{y z}\right)}{\left(\Psi_{y}^{2}+\Psi_{z}^{2}\right)^{3 / 2}}
$$

Where $\Psi_{y}$ and $\Psi_{y y}$ represents the first and second order derivatives of $\Psi$ similarly $\Psi_{z}$ and $\Psi_{z z}$ are the same with respect to z.

The level set function after a small interval $\delta_{t}$ is approximated by eq.(5)

$\Psi_{t}=\alpha *$ Mean curvature $+\frac{f_{0}}{\max \left|f_{0}\right|}$

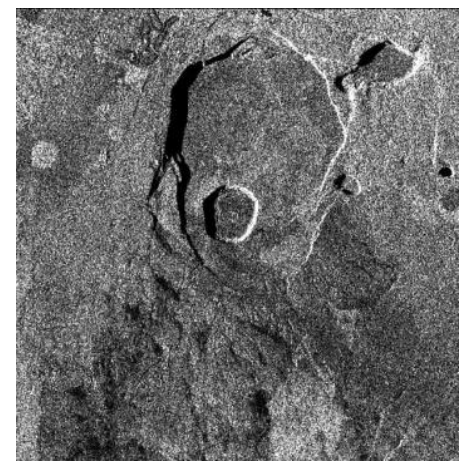

a)Synthetic Aperture Sonar Image

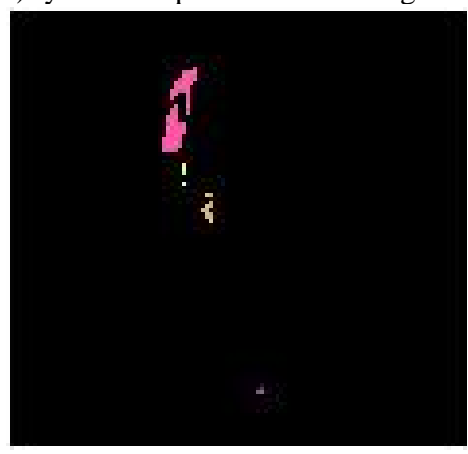

d) Result of counting objects

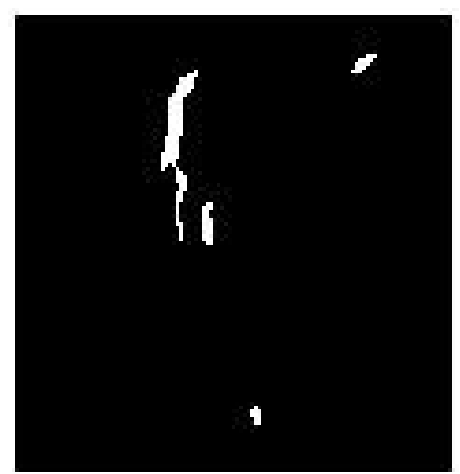

b)Binary mask of Image

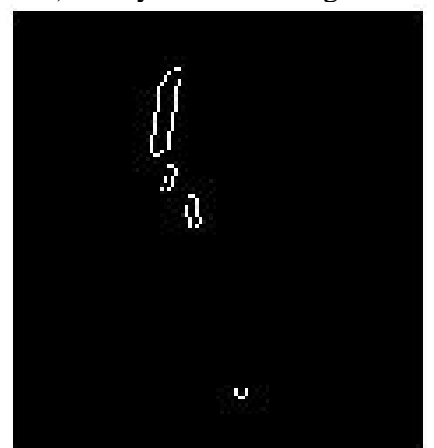

e)Result of Edge Detected Image

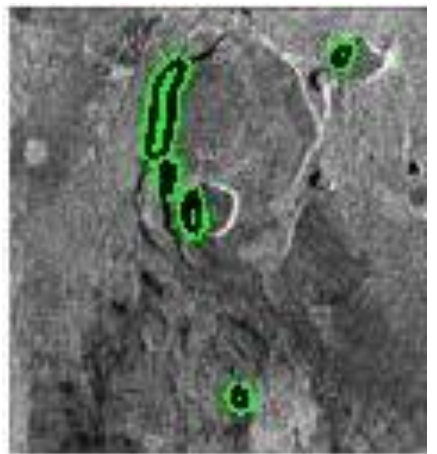

c)Area of Extracted Objects

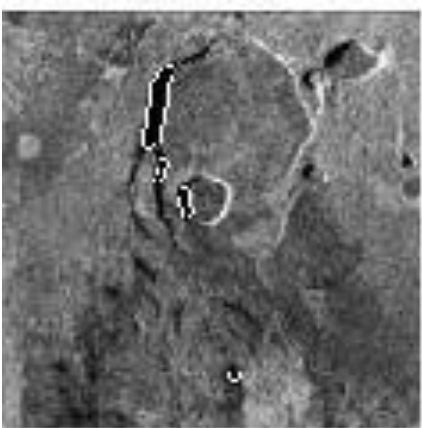

f)Overlaid on Original Image

Fig.1. SAR Image after Segmentation operation by Hybrid region based active contour method can be varied and the inertial influence value will be slightly

\section{Proposed Hybried Paritical Swam Optimi- zation (PSO)}

Eberhart and Kennedy have developed the original PSO in 1995.Swarm intelligence, basic concept of PSO [19] [20]. Proposed hybrid PSO is the combination of basic PSO,DPSO and Fractional Order PSO. In which several swarms using Darwin's survival of the fittest principle and convergence of control depending on fractional calculus. It enhances the ability of PSO algorithm. It will escape from local minima by running the different PSO having different swarms. The searching area is discarded and another area is searched when it is optimized. By this approach at each step swarm extend the particle life and delete the particles.Griinward-Letnico FC defines the fractional differential concept with $\alpha$, but it should be $0 \leq \alpha \leq 1$ to control the particle convergence rate.Each particle $P$,with each swarm $S$ move in multidimensional space according to the $\operatorname{space}\left(F_{p}[t]\right), 0 \leq F_{p}[t] \leq L-1$, and velocity $v_{p}[t]$.The velocity and position moves are highly dependent on local best $\left.(F \dot{[} t]_{p}\right)$ and global best $\left.(G \dot{[} t]_{p}\right)$. According to this local best and global best, the coefficients $w, \rho_{1}$ and $\rho_{2}$ controls the inertial influence when the new

velocity is determined. Depending on the different values of "cognitive" and "social" components $\rho_{1}$ and $\rho_{2}$, the results velocity and position vector are defined as

$$
\begin{aligned}
& v_{p}^{s}[t+1]=\alpha v_{p}^{s}[t]+\frac{1}{2} v_{p}^{s}[t-1]+\frac{1}{6} \alpha(1-\alpha) v_{p}^{s}[t-2]+ \\
& \left.\left.\frac{1}{24} \alpha(1-\alpha)(2-\alpha) v_{p}^{s}[t-3]+\rho_{1} r_{1}(G \dot{[} t]_{p}{ }^{s}-F \dot{[} t\right]_{p}{ }^{s}\right)+ \\
& \left.\left.\left.\rho_{2} r_{2}(G \dot{[} t]_{p}{ }^{s}-F \dot{[t}\right]_{p}{ }^{s}\right) G \dot{[} t\right]_{p}{ }^{s},\left|v_{p}^{s}[t+1]\right| \leq \Delta v \\
& F_{p}[t+1]^{s}=F_{p}[t]+v_{p}^{s}[t+1], 0 \leq F_{p}[t] \leq L-1
\end{aligned}
$$

Weights and mean value of components are calculated using the value of $F_{p}[t+1]^{s}$ when pixels of the image divided into $m$ classes

$$
\begin{gathered}
\text { The weights } w_{i}^{c}=\left\{\begin{array}{c}
\sum_{n=0}^{t_{i}^{c}} p_{i}^{c}, i=1 \\
\sum_{n=t_{i-1}^{c}+1}^{t_{i}^{c}} p_{i}^{c}, 1 \leq i \leq m \\
\sum_{n=t_{i-1}^{c}+1}^{L-1} p_{i}^{c}, i=m
\end{array}\right. \\
\text { and the mean values } \mu_{i}^{c}=\left\{\begin{array}{c}
\sum_{n=0}^{t_{i}^{c}} \frac{p_{i}^{c}}{w_{i}^{c}}, i=1 \\
\sum_{n=t_{i-1}^{c}+1}^{t_{i}^{c}} \frac{p_{i}^{c}}{w_{i}^{c}}, 1 \leq i \leq m \\
\sum_{n=t_{i-1}^{c}+1}^{L-1} \frac{p_{i}^{c}}{w_{i}^{c}}, i=m
\end{array}\right.
\end{gathered}
$$


An efficient method for getting the optimum threshold value is that maximizes the between class variance of each component defined as

$$
\sigma_{R}^{c^{2}}=\sum_{i=1}^{m} w_{i}^{c}\left(\mu_{i}^{c}-\mu_{T}^{c}\right)^{2}
$$

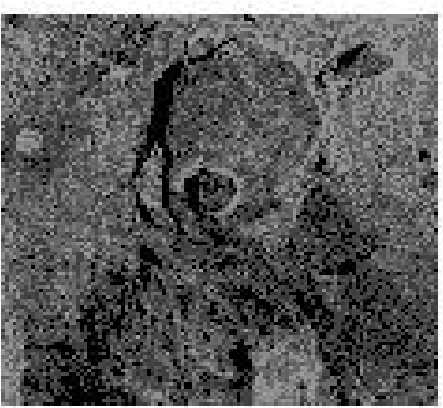

a)Synthetic Aperture Sonar Image

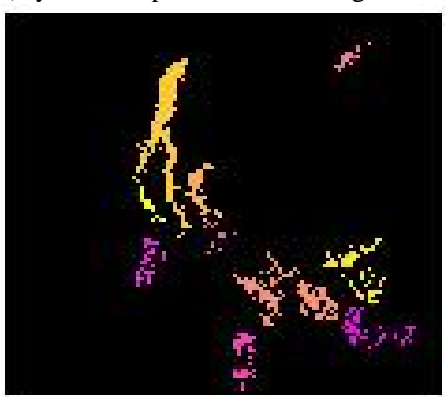

d) Result of counting objects

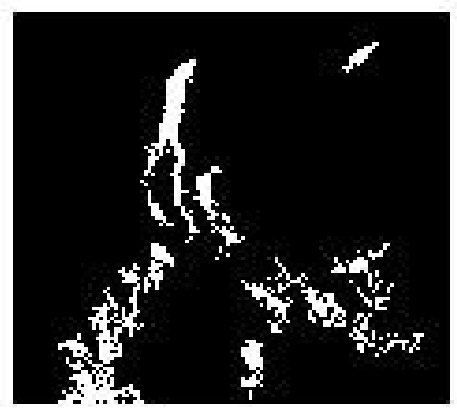

b)Binary mask of Image

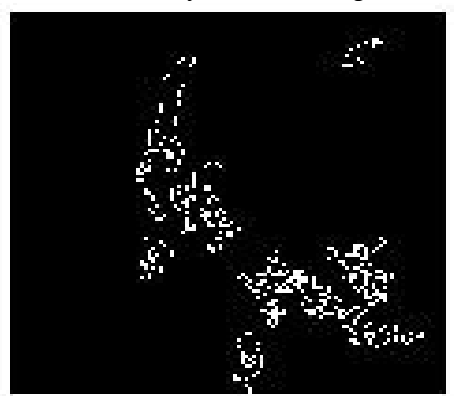

e)Result of Edge Detected Image
The optimum solution to find the thresholds that maximizes the fitness function of each image component is

$$
\emptyset^{c}=\underset{1<t_{i}^{c} \ldots \ldots<t_{n-1}^{c}<L-1}{\operatorname{Max} \sigma_{R}^{c^{2}}\left(t_{i}^{c}\right)}
$$

Based on this optimum solution the swarms fitness may increase or decrease.

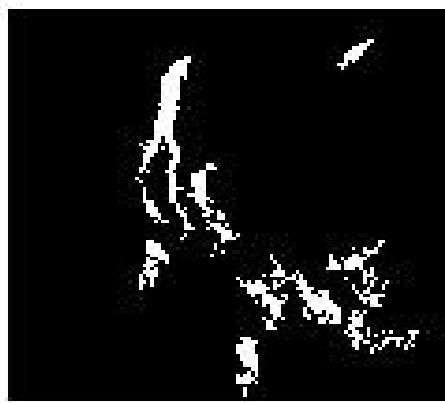

c)Area of Extracted Objects

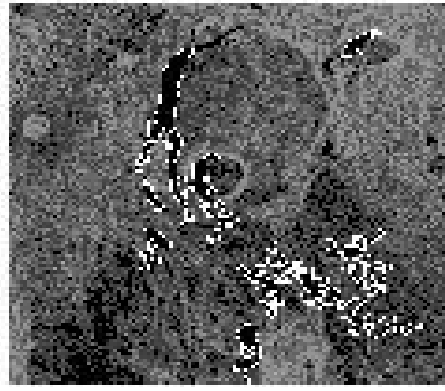

f)Overlaid on Original Image

Fig.2. SAR Image after Segmentation operation using proposed Hybrid PSO Method

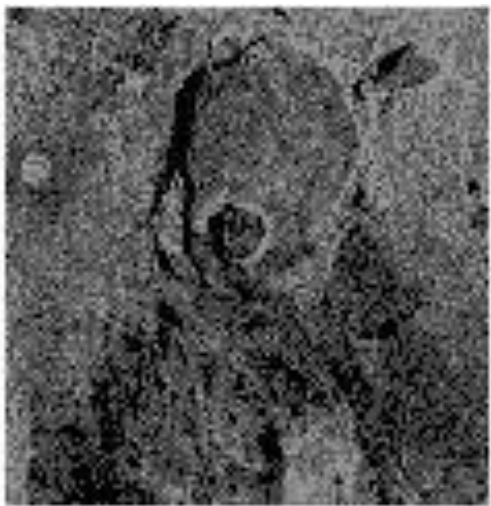

a)Synthetic Aperture Sonar Image

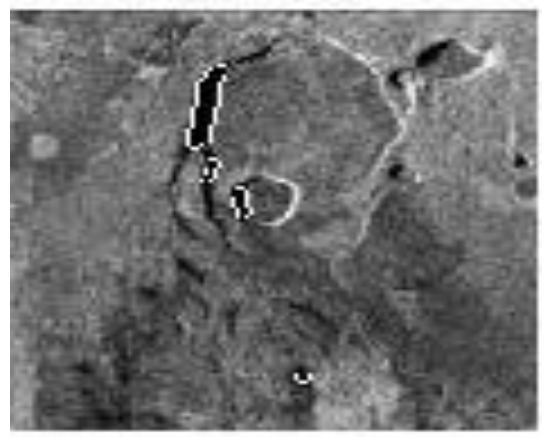

d)Segmented by region based active contour

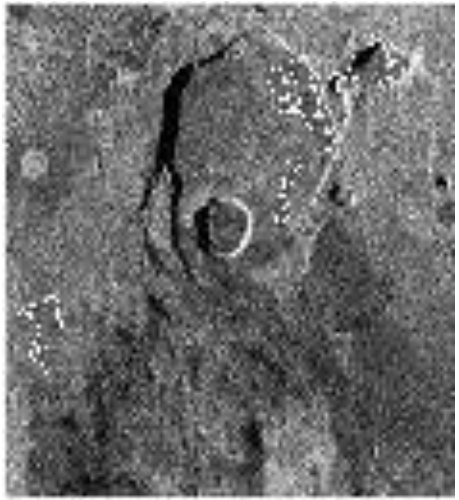

b)Segmented by Otsu Method

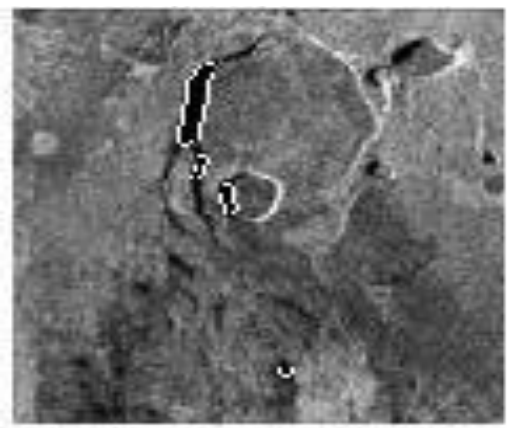

e)Segmented by Active contour Modified Otsu Method

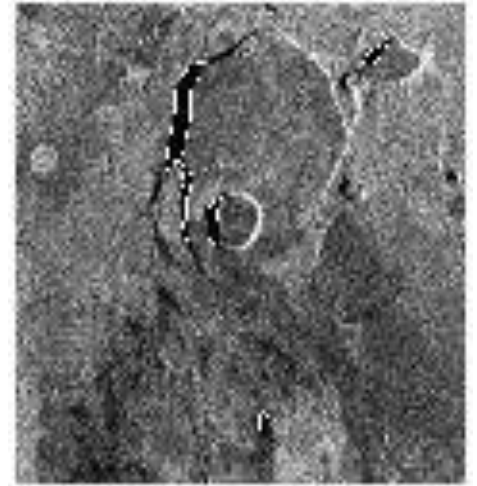

c)Segmented by Modified Otsu Method

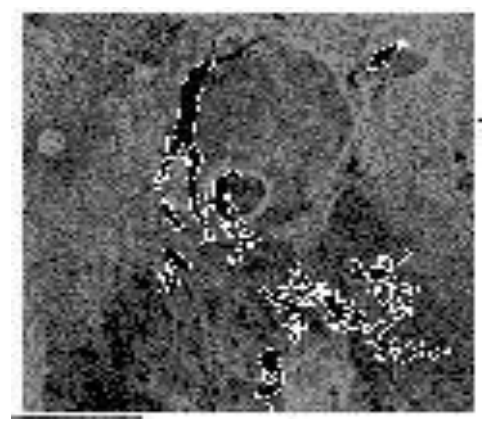
f)Segmented by Proposed Method

Fig. 3: SAR Image after segmentation operation using various techniques.

The Objects extracted after completion of the number of iterations. Fig. 2(c) represents the extracted regions after inserting and removing the swarm particles. This technique identifies the effective regions where the area is around hundreds of meters. The proposed method effectively extracted the objects from a complex 
condition with lee time through more accuracy that matched better with prediction by human experts.

Fig. 3(a)-(f) represents the different methods performs the segmentation process of extraction of objects appearing in an image.

\section{Classification}

Here Fig.4presents the results of a number of images which are under tested with different algorithms. Here the figures are of different categories like sonar image, volcano effected areas and Original Image

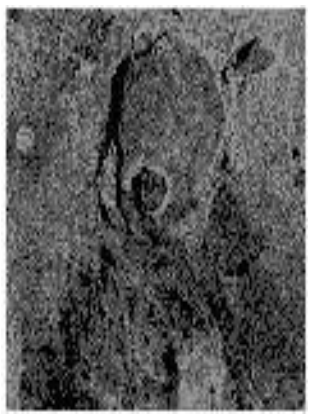
Segmentation Method Conventional Otsu

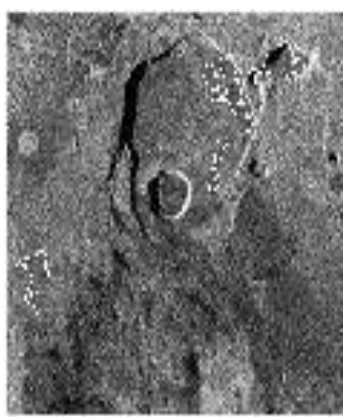
Active
Otsu
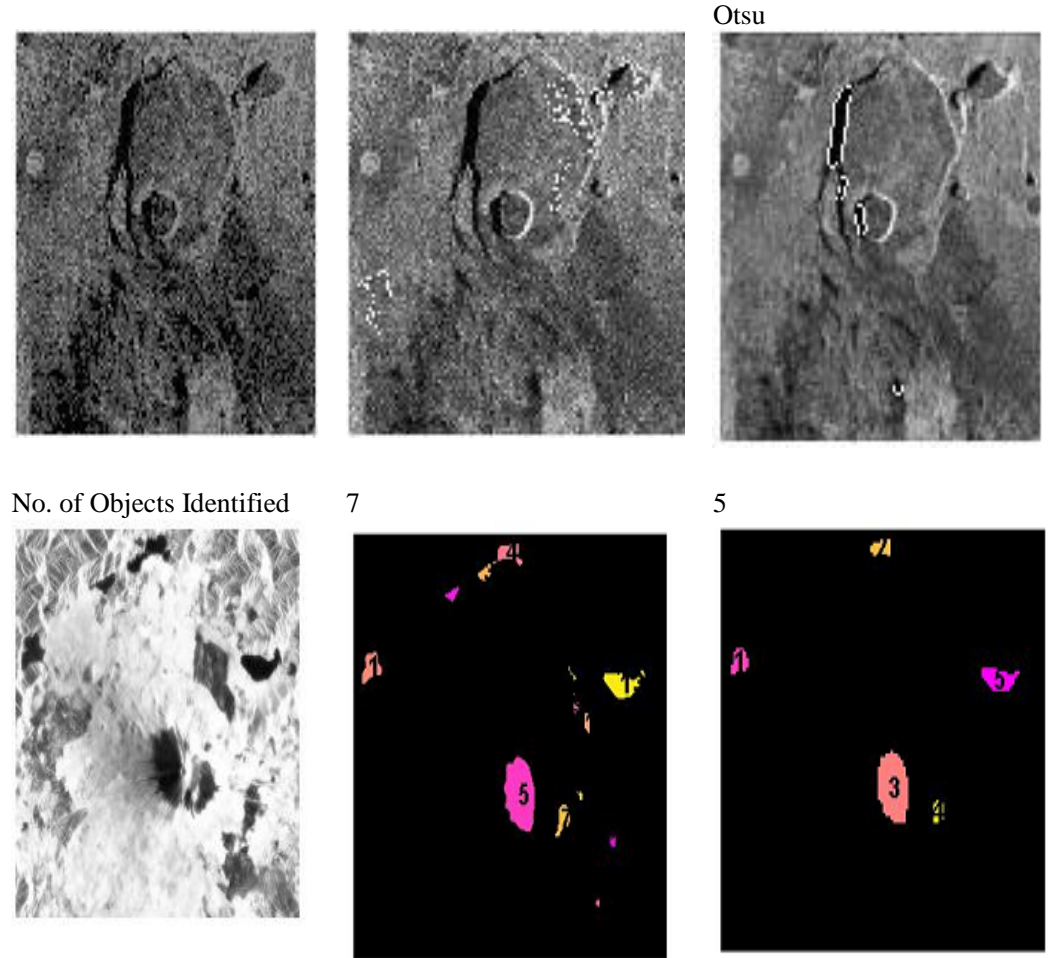

14
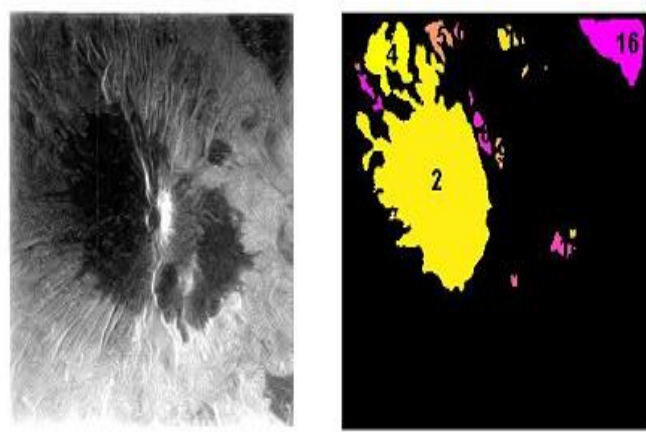

No. of Objects Identified

No. of Objects Identified
6

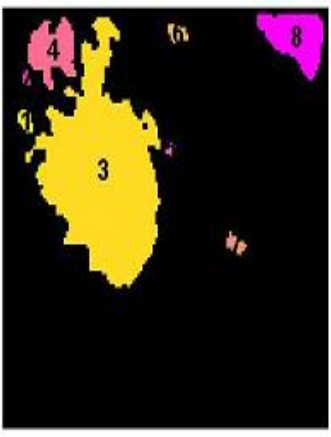

terrain regions. All the methods Conventional Otsu, Active contour modified Otsu presents the unmatched count and location of objects, regions of testing images. But by proposing method the calculated number, location and boundary of the regions extracted accurately. During a training phase the image characteristics are analyzed and the classifier organizes the data into different tables. In the testing phase all the features are calculated. On the basis of the size of the object or the region and location, the observer may come in to conclusion about the area.
Proposed Method

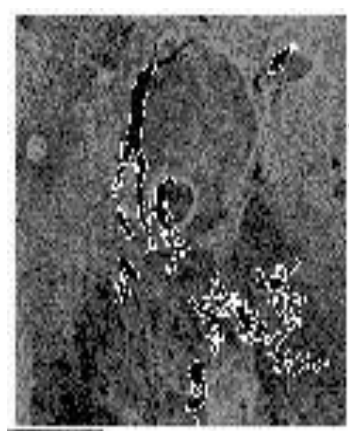

10

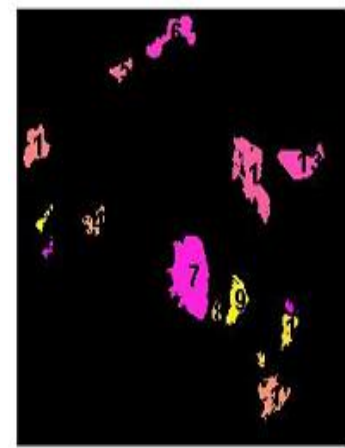

15

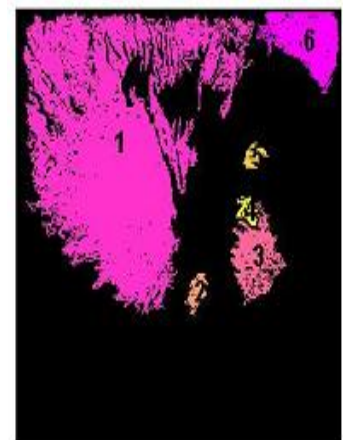

Manual detection

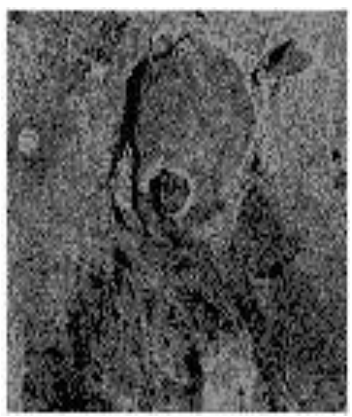

15

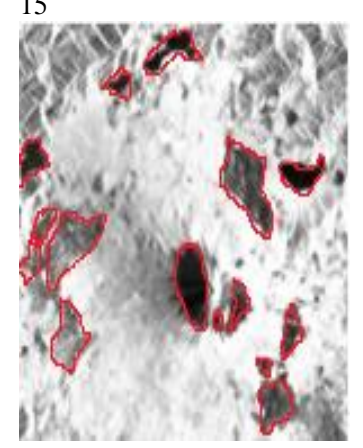

15

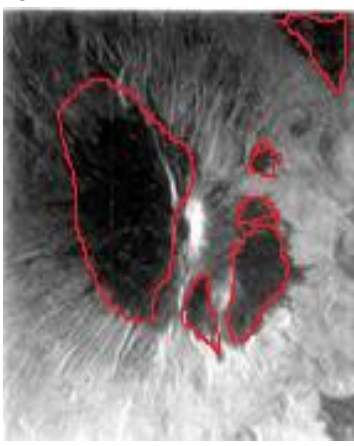

6

region extraction using various techniques like conventional Otsu, Active contour modified Otsu and proposed method with three different images.

Table.1 Numerical Analysis of Regions Extraction using the conventional Otsu method

\begin{tabular}{|c|c|c|c|c|c|c|c|c|}
\hline Method & $\begin{array}{l}\text { Individual } \\
\text { Objects }\end{array}$ & Area & Perimeter & Major Axis & Minor Axis & Eccentricity & Extent & Circularity \\
\hline \multirow{7}{*}{$\begin{array}{l}\text { Otsu Meth- } \\
\text { od }\end{array}$} & 1 & 402 & 151.7817 & 38.53497 & 20.38837 & 0.848567 & 0.386538 & 0.219279 \\
\hline & 2 & 99 & 44.62742 & 13.46642 & 10.8128 & 0.596053 & 0.6875 & 0.624657 \\
\hline & 3 & 180 & 107.9828 & 41.6016 & 8.262782 & 0.980077 & 0.352941 & 0.193987 \\
\hline & 4 & 54 & 43.55635 & 11.27192 & 8.156903 & 0.690169 & 0.613636 & 0.357685 \\
\hline & 5 & 62 & 34.14214 & 12.86287 & 7.524768 & 0.811034 & 0.574074 & 0.668375 \\
\hline & 6 & 326 & 129.6396 & 39.96713 & 13.29896 & 0.943016 & 0.379953 & 0.243754 \\
\hline & 7 & 498 & 154.0244 & 45.23003 & 21.30615 & 0.8821 & 0.365103 & 0.263791 \\
\hline
\end{tabular}


Table.2 Numerical Analysis of Regions Extraction using Active Contour Modified Otsu Method

\begin{tabular}{|c|c|c|c|c|c|c|c|c|}
\hline Method & $\begin{array}{l}\text { Individual } \\
\text { Objects }\end{array}$ & Area & Perimeter & Major Axis & Minor Axis & Eccentricity & Extent & Circularity \\
\hline \multirow{5}{*}{$\begin{array}{l}\text { Active Con- } \\
\text { tour Modified } \\
\text { Otsu Method }\end{array}$} & 1 & 486 & 137.4975 & 62.81852 & 11.51327 & 0.983061 & 0.380878 & 0.32304 \\
\hline & 2 & 135 & 87.45584 & 43.12285 & 5.736158 & 0.991113 & 0.421875 & 0.221802 \\
\hline & 3 & 131 & 60.62742 & 27.40921 & 7.177162 & 0.965108 & 0.606481 & 0.447861 \\
\hline & 4 & 60 & 29.89949 & 12.6588 & 6.265193 & 0.868934 & 0.769231 & 0.8434 \\
\hline & 5 & 72 & 37.45584 & 17.31972 & 5.536591 & 0.947529 & 0.428571 & 0.644916 \\
\hline
\end{tabular}

Table.3 Numerical Analysis of Regions Extraction using the Proposed Method

\begin{tabular}{|c|c|c|c|c|c|c|c|c|}
\hline Method & Follicle & Area & Perimeter & Major Axis & Minor Axis & Eccentricity & Extent & Circularity \\
\hline \multirow{10}{*}{$\begin{array}{c}\text { Proposed } \\
\text { Method }\end{array}$} & 1 & 1026 & 469.2447 & 118.7789 & 26.48742 & 0.974819 & 0.249392 & 0.058554 \\
\hline & 2 & 217 & 188.4092 & 33.24985 & 16.3916 & 0.870039 & 0.339063 & 0.076818 \\
\hline & 3 & 100 & 81.84062 & 28.65072 & 6.331731 & 0.975274 & 0.235294 & 0.187617 \\
\hline & 4 & 267 & 178.4092 & 42.581 & 16.1844 & 0.924952 & 0.283139 & 0.105411 \\
\hline & 5 & 116 & 131.4386 & 22.45817 & 16.43681 & 0.681428 & 0.292929 & 0.084377 \\
\hline & 6 & 286 & 172.267 & 39.78628 & 13.62187 & 0.939563 & 0.488889 & 0.121108 \\
\hline & 7 & 730 & 591.5534 & 65.4821 & 35.43656 & 0.840917 & 0.226427 & 0.026215 \\
\hline & 8 & 393 & 472.4996 & 48.2703 & 30.40607 & 0.776666 & 0.209043 & 0.022121 \\
\hline & 9 & 134 & 91.63961 & 26.40892 & 7.791983 & 0.955481 & 0.265873 & 0.200516 \\
\hline & 10 & 282 & 358.9605 & 49.63963 & 21.43337 & 0.901979 & 0.218944 & 0.027502 \\
\hline
\end{tabular}

Human experts identify the number of objects and affected regions and area of the affected regions shown in Fig. 4.The tables I-III represents the testing results of Sonar image and its detected object features.

\section{Experiment Results}

In the proposed segmentation methods are evaluated by denoised image. The implementation and performance results of different segmentation algorithms are shown in Fig. 3 including conventional Otsu, Modified conventional Otsu. TheRegion based active contour technique generated better results than previous methods. Finally, the proposed Hybrid PSO method extracts the different regions in the image shown in Fig. 2.The proposed methods give the effective results in terms of all the features of extracting objects which can match the information identified by human experts.

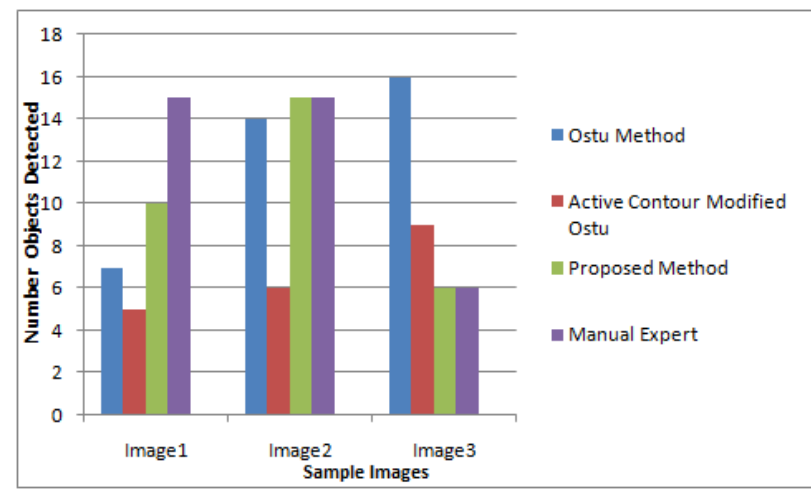

Fig. 5.Illusraion of various techniques in the task of target identification in SAR processing

From the experimental results as consolidated in comparison chart of Fig. 5.Itis observed that our proposed algorithm gives the best result over the existing algorithms and assessment by human experts. Thus, it justifies that the proposed algorithm offers more accurate and automatic detection of objects or regions in SAR images.

\section{Conclusion}

This paper presents the efficient system that automatically detects the objects and segmented areas acquired in SAR images. The SAR image is efficiently segmented for detecting the special regions by hybrid PSO algorithm. This proposed system performs more speed and accuracy to meet the specific requirements. Therefore, this automatic detecting system embedded with Geo graphic analyzer that avoids the painful observation of scientists, extracted object or region features more precisely. Hence the proposed sys- tem is an efficient technique that it will use full for analyzing the SAR image to study the ground condition, icebergs sizes in the sea etc.

\section{References}

[1] Yongjian Yu, Acton, S.T.” Speckle reducing anisotropic diffusion"IEEE Transactions on Image processing,volume 11,issue 11,November 2002.

[2] Junling Zhu; Jianguo Wen; YafengZhang"A new algorithm for SAR image despeckling using an enhanced Lee filter and median filter'IEEE Conference Publications Image and signal processing, Volume: 01,Pages: 224 - 228,2013

[3] Akl, Tabbara, K,Yaacoub.C" An enhanced Kuan filter for suboptimal speckle reduction" Advances in Computational Tools for Engineering Applications (ACTEA), Pages: 91 - 95,2012

[4] T.C.Aysal,K.E.Barner,'Rayleigh maximum like hood Filtering for speckle reduction of Ultrasound Images"IEEE transactions on Medical Imaging,vol.26.no.5,pp.712-727,2007.

[5] Firoju,Loana,CorinaNafomita,J-M Boucher and AlexandruIsar "Image Denoising using new implementation of the hyper analytic wavelet transform"IEEEtaransactions on Instrumentation and Measurement,vol 58,no.8,pp.2410-2416,August 2009.

[6] Mencatti-

ni,Arianna,MarcelloSalmeri,RobertoLojacono,MarcoFrigerio and Federica Caselli "Mammographic images enhancement and denoising for breast cancer detection using dyadic wavelet processing",IEEE Transaction on Instrumentation and Measurement,vol.57,no.7,pp.1422-1430,july 2008.

[7] F.Russo"An Image Enhancement system based on noise estimation"IEEE Transaction on Instrumentation and Measurement,Vol.56,no.4,pp.1435-1442,August 2007.

[8] Gifani,P.H.Behnam and Z.A.Sani "Noise reduction of ECG Images based on temporal information"IEEE Transaction on Ultrasonic, Ferroelectrics and frequency Control,vol.61,no.4,pp.620630,April 2004

[9] W. X. Kang, Q. Q. Yang, R. R. Liang ,"The Comparative Research on Image Segmentation Algorithms", IEEE Conference on ETCS, pp. 703-707, 2009

[10] Er. Nirpjeetkaur and ErRajpreeetkaur, "A review on various method of image thresholding",IJCSE-2011.

[11] ZhongQuandLiHang"Research on Iimage Segmentation Based on the Improved Otsu Algorithm.,"2010

[12] W. X. Kang, Q. Q. Yang, R. R. Liang "The Comparative Research on Image Segmentation Algorithms", IEEE Conference on ETCS, pp. 703-707, 2009.

[13] Z. Ningbo, W. Gang, Y. Gaobo, and D. Weiming, "A fast 2d otsuthresholding algorithm based on improved histogram," in Pattern Recognition, 2009. CCPR 2009. Chinese Conference on, 2009, pp. 1-5.

[14] T.Chan and L.Vese,"Active contours with outedges"IEEE Transactions Image Processing,vol.10,issue 2,pp.266-277,2001..

[15] S.-C. Zhu and A. Yuille, "Region competition: Unifying snakes, regiongrowing, and Bayes/MDL for multiband image segmentation," IEEETrans. Pattern Anal. Mach. Intell., vol. 18, no. 9, pp. 884-900, Sep.1996. 
[16] S. Kichenassamy, A. Kumar, P. Olver, A. Tannenbaum, and A. Yezzi, "Gradient flows and geometric active contour models," in Proc. 5th Int.Conf. Comput. Vis., 1995, pp. 810-815.

[17] R. Ronfard, "Region-based strategies for active contour models," Int.J. Comput. Vis., vol. 13, no. 2, pp. 229-251, Oct. 1994.

[18] C. Samson, L. Blanc-Feraud, G. Aubert, and J. Zerubia, "A variationalmodel for image classification and restoration," IEEE Trans. PatternAnal. Mach. Intell., vol. 22, no. 5, pp. 460-472, May 2000.

[19] J. Kennedy, and R. Eberhart, Swarm Intelligence, San Francisco: Morgan Kaufmann Publishers, 2001.

[20] Gao,Hao,WenboXu,Junsun and Yulan Tang" Multilevel thresholding for image segmentation through an improved quantumbehaved particle swarm algorithm "IEEE Transactions on Instrumentation and Measurement,vol.59,no.4,pp.934-946,April 2010.

[21] Y. zhiwei, C. hongwei, L wei and Z. jinping, "Automatic threshold selection based on Particle Swarm Optimization algorithm," in the proceedings International Conference on Intelligent Computation Technology and Automation, pp. 36-39, 2008

[22] C. Wei and F. Kangling, "Multilevel Thresholding Algorithm Based on Particle Swarm Optimization for Image Segmentation," in the Proceedings of the 27th Chinese Control Conference, July 16-18, Kunming, Yunnan, China, pp. 348-351, 2008

[23] P. D. Sathya, R. Kayalvizhi, "PSO-Based TsallisThresholding Selection Procedure for Image Segmentation," International Journal of Computer Applications, Vol. 5, No. 4, pp. 39-46, 2010

[24] T. Hongmei, W. Cuixia, H. Liying, and W. Xia, "Image Segmentation Based on Improved PSO," the proceedings of the International Conference on Computer and Communication Technologies in Agriculture Engineering(CCTAE2010), pp. 191-194, 2010.

[25] Y. Shi, and R. Eberhart, "A modified particle swarm optimizer," in the Proceedings of the IEEE International Conference on Evolutionary Computation, Piscataway, NJ, pp. 69-73, 1998.

[26] Liu J., Yang Y. H., "Multiresolution Color Image Segmentation," IEEE Trans. on PAMI, Vol. 16, No. 7, pp. 689-700, 1994.

[27] E.J.S. Pires, J.A.T. Machado, P.B.M. Oliveira, J.B. Cunha, L. Mendes, "Particle swarm optimization with fractional-order velocity," Journal on Nonlinear Dynamics, vol. 61, pp. 295301,2010 .

[28] J. Fan, M. Han, and J. Wang, "Single point iterative weighted fuzzy C-means clustering algorithm for remote sensing image segmentation," Pattern Recognition, vol. 42 (2009), pp. 2527 2540, 2009.

[29] K. Hammouche, M. Diaf, and P. Siarry, “A comparative study of various meta-heuristic techniques applied to the multileve thresholding problem," Engineering Applications of Artificial Intelligence, vol. 23 (2010), pp. 676-688, 2010.

[30] M. Jiang, Y.P. Luo, and S.Y. Yang, "Stochastic convergence analysis and parameter selection of the standard particle swarm optimization algorithm," Information Processing Letters, vol. 102, no. 1, pp. 8-16, 2007

[31] K. Yasuda, N. Iwasaki, G. Ueno, and E. Aiyoshi, "Particle Swarm Optimization: A Numerical Stability Analysis and $\mathrm{Pa}$ rameter Adjustment Based on Swarm Activity," IEEJ Transactions on Electrical and Electronic Engineering, Wiley InterScience, vol. 3, pp. 642-659, 2008.

[32] Md Zia Ur Rahman, B.Malakonda Reddy, "Efficient SAR Image Segmentation Techniques using Biasfield Estimation",

Journal of Scientific and Industrial Research, vol. 76, pp. 335338, 2017.

[33] M.L.M. Lakshmi, K.Rajkamal, S.V.A.V.Prasad, Md.Zia Ur Rahman, "Amplitude Only Linear Array Synthesis With Desired Nulls Using Evolutionary Computing Technique", The Applied of Computational Electromagnetics Society Journal, vol.31, no.11, pp. 1357-1361, November, 2016

[34] P.V.V. Kishore, A.S.C.S. Sastry, Md. Zia Ur Rahman, "Double Technique for Improving Ultrasound Medical Images", Journal of Medical Imaging and Health Informatics, vol.6, no.3, pp.667675, 2016.

[35] M. Lakshmi, Md Zia Ur Rahman, "Efficient Speckle Noise Reduction Techniques for Synthetic Aperture Radars in Remote Sensing Applications", International Review of Aerospace Engineering Vol.9, no.10, 2016, pp.114-122.

[36] M. Lakshmi, Md Zia Ur Rahman, "Analysis of Synthetic Aperture Radar Images using Brute Force Thresholding and Gradient Guide Filters", Journal of Theoretical and Applied Information Technology,Vol.93, no.1, 2016, pp.152-163.
[37] B. Mala Konda Reddy, Md. Zia Ur Rahman, "Novel Segmentation Technique for Target Tracking in Synthetic Aperture Radars", International Journal of Control Theory and Applications,Vol.10, no.35, 2017, pp.335-341.

[38] Y. Murali Krishna, Md. Zia Ur Rahman and Dr B.V. Rama Mohana Rao, "Beam Steering in Smart Antennas using an Efficient Adaptive Signal Processing Algorithm," International Journal of Research and Reviews in Signal Acquisition and Processing, Vol 1(3), Sep. 2011.

[39] K. Murali Krishna, Md. Zia Ur Rahman, "Lung Parenchyma Detection using Levelset Segmentation", International Journal of Control Theory and Applications, Vol.10, no.35, 2017, pp.207215.

[40] K. Sarath Kumar, Md. Zia Ur Rahman, "A New Computation Method for Pointing Accuracy of Cassegrain Antenna in Satellite Communication", Journal of Theoretical and Applied Information Technology,Vol.95, no.13, 2017, pp.3062-3074

[41] Md. Zia Ur Rahmna, K. Murali Krishna, "Efficient Adaptive Beamforming Algorithms for Smart Antennas", International Journal of Control Theory and Applications, Vol.10, no.35, 2017, pp.173-181.

[42] Md. Zia Ur Rahman, et al., "A Low Complex adaptive algorithm for Antenna beam steering", IEEE 2011 International Conference on Signal Processing, Communications, Computing and Networking Technology (ICSCCN 2011), ISBN: 978-1-61284-6538, pp.317-321, 2011. 